\title{
Simulations of powerful microwave oscillators with oversized electrodynamics systems
}

\author{
V.Yu. Zaslavsky and N.S. Ginzburg
}

Institute of Applied Physics of the Russian Academy of Sciences, Nizhny Novgorod, Russia, zas-vladislav@appl.sci-nnov.ru

\section{Introduction}

Nowadays, various types of numerical codes are effective tools for analysis and optimization of electron microwave devices. Special codes are widely used to simulate the microwave components, the electron-optical system, and nonlinear dynamics. Nevertheless, up to now most of the simulations related to electron optics and electron-wave interaction were restricted by exploiting 2D self-consistent numerical models. At the same time, the state-of-the-art high-performance computational clusters combined with modern commercial codes look sufficient for 3D physical modeling of vacuum electronic devices with strongly oversized interaction space.

The paper is devoted to review of recent results of the CST STUDIO SUITE particle-in-cell (PIC) simulations of broad class of relativistic and sub relativistic microwave oscillators and amplifiers. In this report we consider terahertz-band gyrotrons with conventional cylindrical and planar configurations of the interaction space [1-3]. Here we discuss generators operating at the fundamental harmonic, as well as at the second and third harmonics. For improvement of stability of high cyclotron harmonic operation regime, multiple-beam schemes of gyrotrons are investigated [2]. Studies of relativistic surface wave Cherenkov oscillators with $1 \mathrm{D}$ and $2 \mathrm{D}$ periodical slow-wave structures were investigated $[4,5]$. For all models comparison with experimental data and results of theoretical consideration based on simplified averaged equations are carried out. This analysis demonstrates that at present stage 3D PIC simulations can be considered as an effective tool for design and development of new schemes of powerful microwave generators and amplifiers. In addition, with the use of modern graphics processing units, it is possible to optimize parameters of electron devices for improvement characteristics within a reasonable calculation time.

\section{High cyclotron harmonic operation in the double-beam THz gyrotrons}

In this section, we explore a double-beam scheme of a short-wavelength gyrotron [2]. To date, the authors have extended this concept to the development of $\mathrm{THz}$ gyrotrons operating in $\mathrm{CW}$ regime at the second cyclotron harmonic. A double-beam scheme of a shortwavelength gyrotron with two generating helical electron beams operating at the second cyclotron harmonic is studied using 3D PIC simulations. Analysis shows that the introduction of an additional generating electron beam allows increase drastically the operating current of a second-harmonic gyrotron with the simultaneous suppression of self-excitation of spurious modes at the fundamental harmonic. This PIC code allows integrating directly (without averaging) the Maxwell equations to- gether with the electron motion equations in the real geometry of the interaction space. The simulation parameters are shown in the figure 1 . For a single-beam scheme current value of $2 \mathrm{~A}$ leads to the simultaneous excitation of the spurious $\mathrm{TE}_{1,4}$ mode at the fundamental harmonic (see Fig. 1b). At the same time, single-mode oscillation with the $\mathrm{TE}_{8,5}$ mode excited at the second harmonic was obtained in the double-beam scheme for a total current of $2 \mathrm{~A}$ (see Fig. 1c). The radiated power of the parasitic modes remains at the noise level. As a result, the radiated power of a double-beam gyrotron exceeds the power of a single-beam gyrotron by a factor of four. The developed concept makes it possible to realize highpower (several hundred watts) single-mode gyrotrons in the $0.7-1.0 \mathrm{THz}$ frequency range.

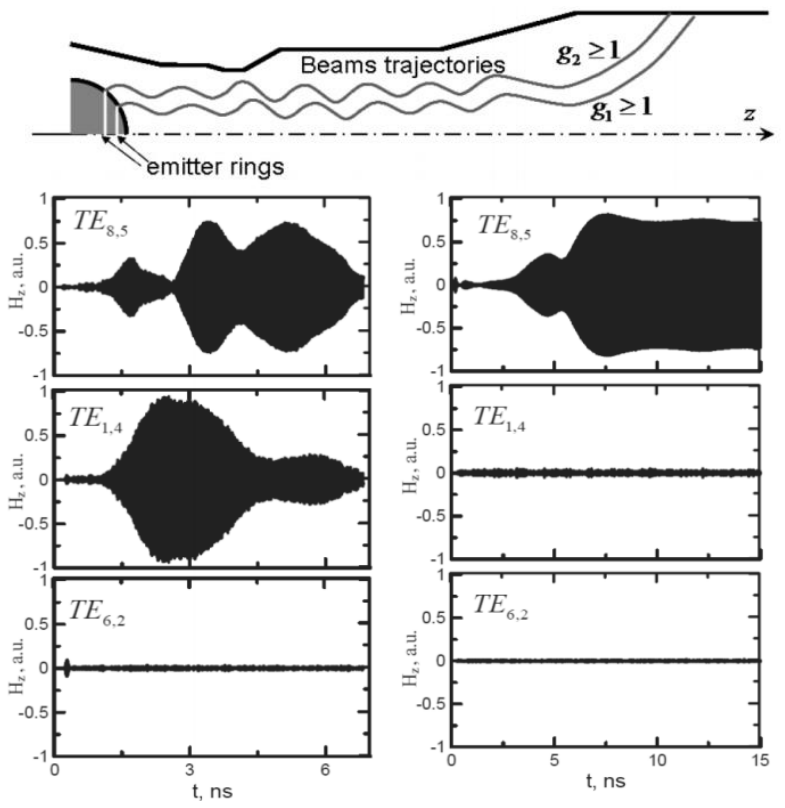

Fig. 1. Scheme of a double-beam $0.78 \mathrm{THz}$ gyrotron with two generating electron beams (top). Temporal evolution of the competing mode amplitudes in the single-beam (left) and doublebeam (right) schemes for $I_{\text {total }}=2 \mathrm{~A}, U=20 \mathrm{kV}, I_{\text {beam } 1}=0.68 \mathrm{~A}$, $I_{\text {beam } 2}=1.32 \mathrm{~A}, g_{1,2}=1.3, R_{\text {beam } 1}=0.46 \mathrm{~mm}, R_{\text {beam } 2}=0.72 \mathrm{~mm}$, $R=3 \mathrm{~mm}, L=12 \mathrm{~mm}$

\section{Powerful high-harmonic THz-range planar gyro- tron with transverse energy extraction}

To increase output power of terahertz gyrotrons to several hundred kilowatts, we suggest using a planar geometry of interaction space with a sheet electron beam and transverse energy extraction [3]. An advantage of this scheme in comparison with conventional cylindrical geometry is the possibility to ensure effective mode selection over the open transverse coordinate in combination with radiation outcoupling that leads to a substantial reduction of Ohmic losses. 


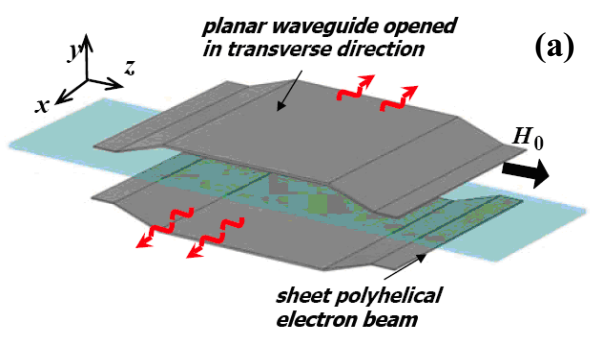

(b)
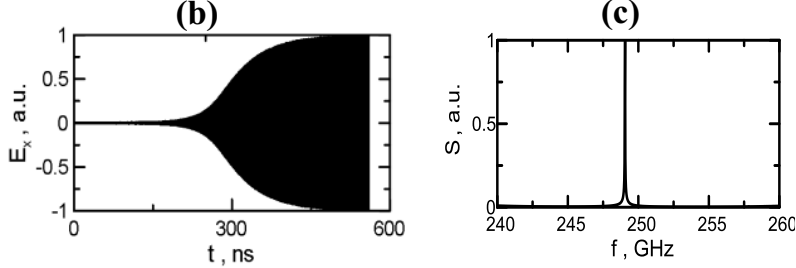

(d)
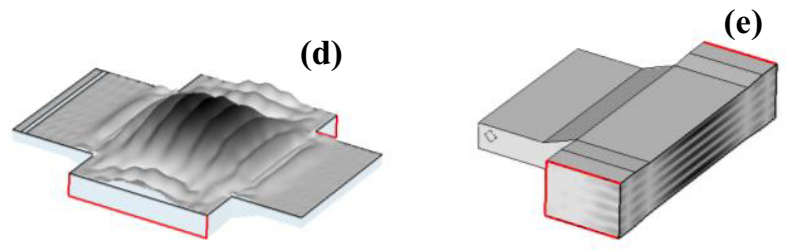

Fig. 2. Results of simulations of planar gyrotron at the third cyclotron harmonic with transverse energy extraction: $(a)$ principal scheme planar gyrotron using in the 3D simulations; (b) establishment of steady state regime of generation for guide magnetic field of $3.35 \mathrm{~T} ;(c)$ radiation spectrum, $(d),(e)$ spatial distributions of excited $\mathrm{TE}_{1,5}$ mode of open planar waveguide; (d) field profile at $(\mathrm{x}, \mathrm{z})$ plane with one variation along both coordinates, $(e)$ transverse structure at $(\mathrm{x}, \mathrm{y})$ plane

Simulations of the nonlinear dynamics of the planar gyrotron at the third cyclotron harmonic were performed for an operating frequency of $250 \mathrm{GHz}$, an accelerating voltage of $80 \mathrm{kV}$, a total current of $15 \mathrm{~A}$, a pitch factor of $g=1$, a distance between plates of $3 \mathrm{~mm}$, and the plate width of $1 \mathrm{~cm}$. At a magnetic field of $3.35 \mathrm{~T}$, the modeling demonstrates establishment of the steady-state regime at the third cyclotron harmonic at the mode with single longitudinal variation and its spatial distribution are shown in Fig. 2. The total efficiency at these parameters was $8 \%$, and the output power $\sim 50 \mathrm{~kW}$.

\section{Oversized Ka-band surface-wave oscillator based on 2D periodical corrugated structure}

To study the possibility of achieving a gigawatt power level, we simulate Ka-band surface wave oscillator (SWO) with 2D corrugated structure of cylindrical geometry based on the "SINUS-6" accelerator (IAP RAS) [5]. This accelerator generates an explosive-emission electron beam with particles energy of $500-700 \mathrm{keV}$, beam current of several kiloamperes and about $20 \mathrm{~ns}$ duration in a single-pulse regime.

Figure 3 shows the results of modeling in the frame of 3D PIC code. For a Ka-band oscillator with a sufficiently large oversized waveguide (perimeter of about 16 wavelengths), a steady-state regime settled with a narrow radiation spectrum shown in Fig. $3 \mathrm{~b}$. In this regime the output radiation pattern is a superposition of azimuthallysymmetric modes of a regular cylindrical waveguide. At the operating frequency of $36 \mathrm{GHz}$, with the total beam current of $4 \mathrm{kA}$ and the particles energy of $0.7 \mathrm{MeV}$, the output power of the simulated oscillator amount to $250 \mathrm{MW}$ with an electron efficiency of about $10 \%$.

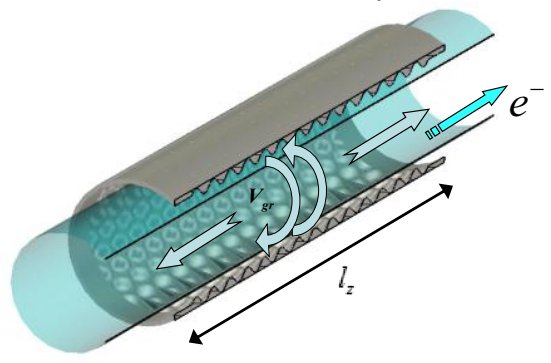

(a)

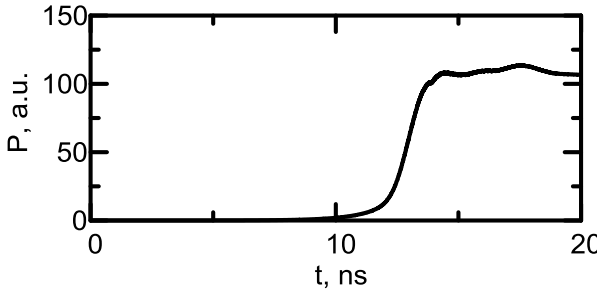

(b)

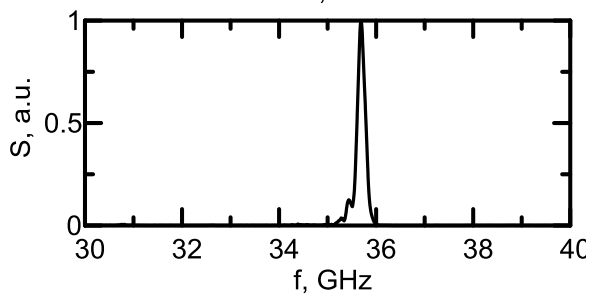

(c)

Fig. 3. Results of simulations of oversized Ka-band SWO with 2D corrugated structure based on "SINUS-6" accelerator: $(a)$ principal scheme of the oscillator; $(b)$ time dependence of the output power and $(c)$ radiation spectrum

\section{Acknowledgments}

This work is partially supported by the Russian Scientific Foundation (grant \#17-19-01605).

\section{References}

1. V.Yu. Zaslavsky, N.S. Ginzburg, M.Yu. Glyavin, et al. Three-dimensional particle-in-cell modeling of terahertz gyrotrons with cylindrical and planar configurations of the interaction space. // Physics of Plasmas. 2013. V. 20. P. 043103.

2. N.S. Ginzburg, M.Yu. Glyavin, A.M. Malkin, et al. Improvement of stability of high cyclotron harmonic operation in the double-beam THz range gyrotrons. // IEEE Transaction on Plasma Science. 2016. V. 44. P. 1303.

3. N.S. Ginzburg, I.V. Zotova, A.S. Sergeev, et al. HighPower Terahertz-Range Planar Gyrotrons with Transverse Energy Extraction. // Physical Review Letters. 2012. V. 108. P. 105101.

4. N.S. Ginzburg, A.M. Malkin, A.S. Sergeev, V.Yu. Zaslavsky. Quasi-optical theory of relativistic surface-wave oscillators with 1D and 2D periodic planar structures. // Physics of Plasmas. 2013. V. 20, P. 113104.

5. N.S. Ginzburg, A.M. Malkin, A.S. Sergeev, V.Yu. Zaslavsky. Oversized co-axial and cylindrical surface-wave oscillators with 2D periodical grating (quasi-optical model). // Journal of Applied Physics. 2013. V. 113. P. 104504. 